\title{
Identifier les problèmes et apporter des solutions
}

\author{
Une première réussie: le premier symposium MedEd de l'ISFM consacré à l'avenir de \\ la formation médicale, qui s'est tenu le 11 septembre 2014 au Kultur Casino à Berne, \\ a rencontré un vif succès.
}

Bruno Kesseli, Isabel Zwyssig

\footnotetext{
* Le rapport peut être téléchargé au format PDF à l'adresse www.bag.admin.ch/ gesundheit2020/index. html?lang=fr
}

Dans son discours d'ouverture, Werner Bauer, président de l'ISFM, a immédiatement souhaité attirer l'attention des participants sur l'essentiel: la formation médicale; ou MedEd, pour Medical Education. Avant d'ajouter non sans fierté: «Si nous pensons que notre système de santé est l'un des meilleurs au monde, alors cela concerne aussi la formation médicale.»

Dans le domaine de la formation postgraduée et continue, Werner Bauer et les collaborateurs de l'ISFM contribuent sans aucun doute au bon fonctionnement de notre système de santé - grâce aussi au premier symposium MedEd, consacré à l'avenir de la formation médicale. Le vif succès remporté par cette manifestation montre clairement que l'initiative du président de l'ISFM répond à un besoin des acteurs concernés de près ou de loin par la formation médicale.

Mais l'ISFM ne se contente pas d'organiser et de gérer la formation postgraduée et continue des médecins. A l'aide de huit exemples, Werner Bauer a brièvement présenté toute l'importance que revêt la qualité de la formation pour l'ISFM. Et dans ce domaine,

bkesseli[at]emh.ch

izwyssig[at]emh.ch

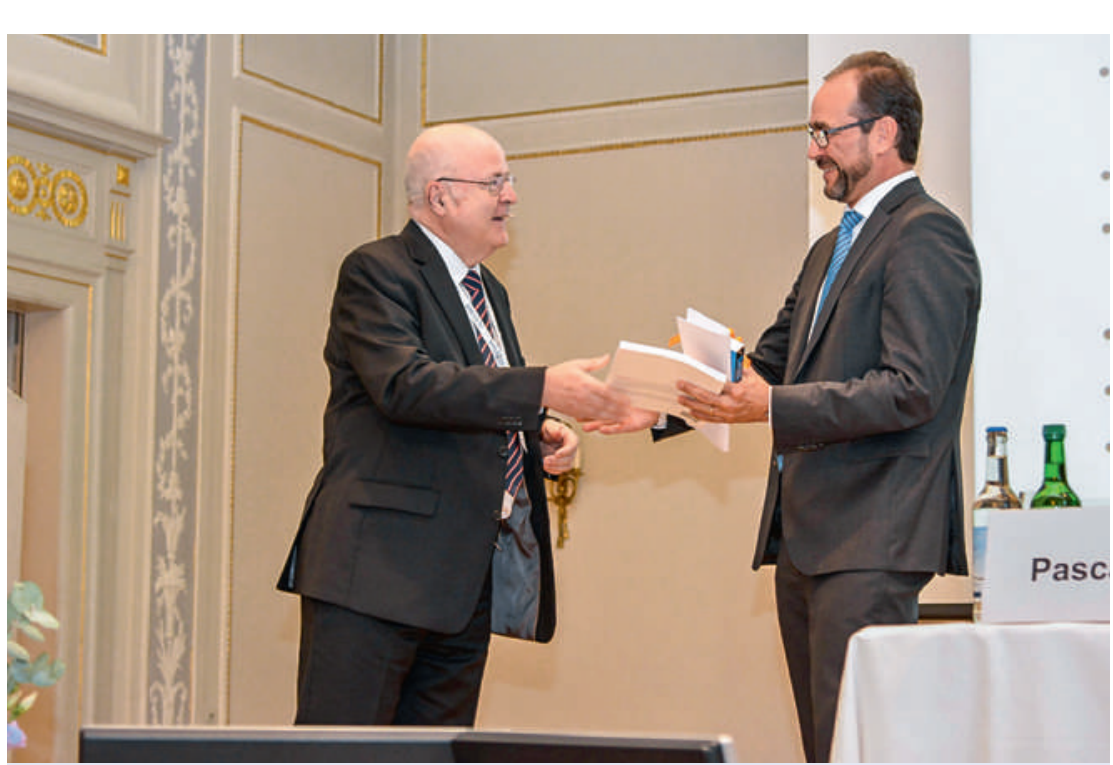

«De nombreux thèmes et intérêts communs»: Werner Bauer, président de l'ISFM (à gauche), et Pascal Strupler, directeur de I'OFSP. Photos Healthworld les défis ne manquent pas. A commencer par le financement, qui n'est toujours pas garanti, en passant par les changements structurels en cours dans le domaine de la santé et au sein de la société (temps partiel, équilibre vie privée / vie professionnelle), aux questions controversées dont celle de la régulation de la formation de spécialiste selon les besoin qualitatifs et quantitatifs du secteur de la santé - les thèmes sont nombreux. En plus d'identifier les problèmes existants, le premier symposium MedEd a également visé à apporter des réponses et à susciter l'échange entre les acteurs issus de différents horizons.

\section{Pascal Strupler: \\ «De nombreux intérêts communs»}

Par sa présence, Pascal Strupler, directeur de l'Office fédéral de la santé publique (OFSP), a souhaité témoigner l'intérêt qu'il porte à la formation médicale et à la collaboration avec l'ISFM. Une collaboration «non triviale», selon lui. Même si le corps médical et l'OFSP ne sont pas toujours sur la même longueur d'onde, ils partagent de nombreux thèmes et intérêts communs.

Constatant que le système de santé est soumis à de profonds changements, notamment d'ordre démographique, le directeur de l'OFSP a tenu à préciser que l'OFSP attache une grande importance à ce que les médecins puissent continuer d'exercer leur profession dans les meilleures conditions. La question de la régulation du nombre de médecins admis à la formation dans les différentes disciplines est en soi particulièrement complexe. Mais il ne faut pas se laisser déstabiliser par cette complexité. En effet, le Conseil fédéral a indiqué dans son rapport «Santé2020» que la garantie de la formation prégraduée, postgraduée et continue revêt une importance essentielle pour la qualité de la prise en charge, l'un des quatre champs d'action prioritaires de sa stratégie. *

P. Strupler a tout particulièrement souligné l'importance croissante de la collaboration interprofessionnelle. Selon lui, il ne fait pas de mystère que l'idée que se font les différents acteurs de la collaboration diffère et que les divergences de vue sont donc inévitables. Mais il s'est néanmoins montré optimiste en estimant que ce processus inéluctable permettra en définitive d'intensifier et d'améliorer la collaboration. 


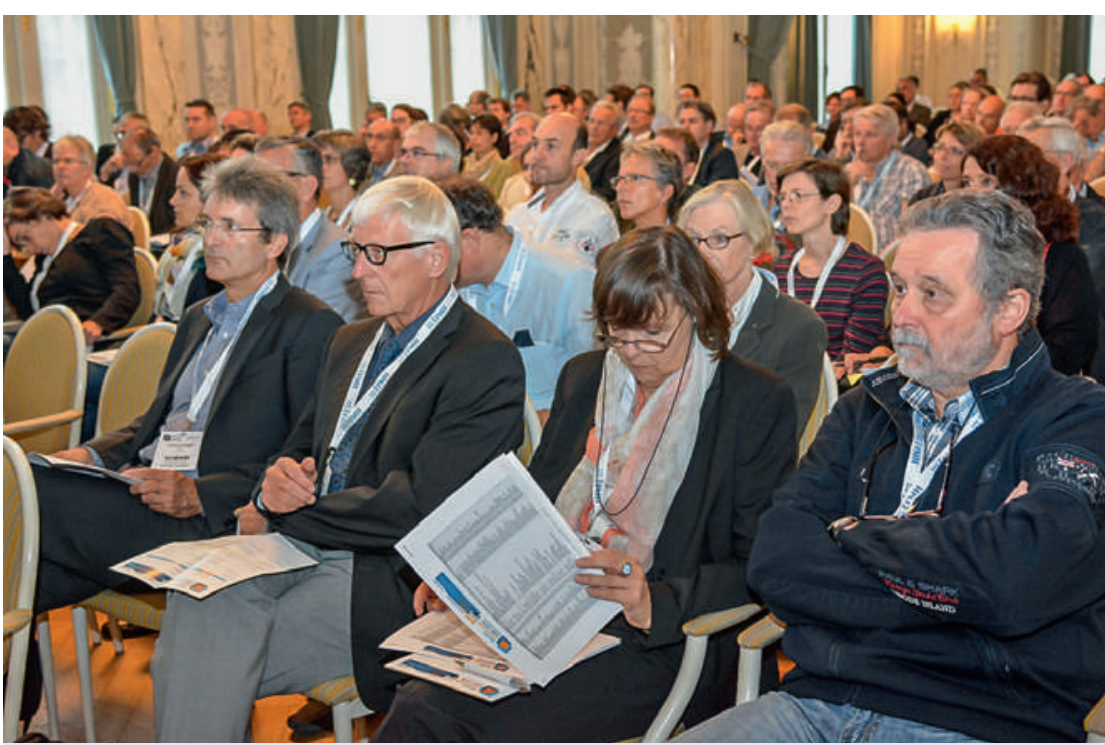

Salle comble: le premier symposium MedEd a rencontré un vif succès.

\section{Werner Kübler: «élargir» les exigences}

Werner Kübler a, quant à lui, consacré son exposé aux «changements structurels à l'hôpital: les conséquences pour la formation postgraduée». Directeur de l'Hôpital universitaire de Bâle, Werner Kübler est également médecin, ce qui lui confère d'autant plus de crédibilité lorsqu'il déclare: «Mon cœur bat au rythme de la formation postgraduée et de la promotion de la relève.» Dans sa fonction de directeur hospitalier, il est actuellement confronté à de nombreux défis qui concernent également la formation postgraduée.

Ces changements structurels et organisationnels sont aussi à l'œuvre au sein des grands hôpitaux comme celui de Bâle. Les cliniques spécialisées dans une seule discipline constituent de plus en plus une exception, et les centres interdisciplinaires la règle. La prise en charge des patients au sein de cliniques spécialisées est progressivement remplacée par une prise en charge axée sur le patient par une équipe interprofessionnelle. S'ajoutent à cela les coopérations multilatérales et la répartition des processus entre différents hôpitaux.

Pour W. Kübler, il est évident que ces changements n'épargnent pas la formation postgraduée des médecins. Les formateurs doivent acquérir de nouvelles compétences afin de les transmettre à leur tour à leurs jeunes confrères, ce qui exige de repenser l'organisation de la formation. S'il ne propose pas de solution miracle, W. Kübler formule néanmoins des thèses intéressantes. Il prévoit notamment un déplacement des pôles d'importance en matière de formation postgraduée, qui pourrait prendre la direction suivante:

- de l'établissement de formation postgraduée (structure) au contenu de la formation;

- de l'établissement de formation postgraduée (lieu) au réseau de formation;
- de l'établissement de formation postgraduée (temps sur place) aux compétences acquises;

- de la formation postgraduée spécialisée (contenu) à la personnalité professionnelle.

\section{Volker Amelung: la régulation est inévitable}

«Réguler la formation postgraduée de spécialiste est-ce pertinent et possible?» C'est à cette question que s'est intéressé Volker Amelung, économiste de la santé de la Haute école de médecine d'Hanovre, dans son exposé. Selon lui, les évolutions depuis les années 90 rendent inévitables une planification et une régulation dans le domaine de la prise en charge médicale, sans quoi il en résultera un décalage entre les différentes disciplines et les différentes régions, comme c'est le cas dans une grande partie de l'Allemagne. Dans le domaine de la santé, l'équilibre entre l'offre et la demande ne se fait pas automatiquement, estime l'économiste.

La mise en œuvre de la planification des soins doit s'inspirer des expériences des autres pays. V. Amelung signale à cet égard l'existence de 18 études portant sur la planification du personnel dans le système de santé. Pour lui, le modèle australien est le plus prometteur. Dynamique, ce modèle prévoit le réexamen régulier des hypothèses et des pronostics sur lesquels il se fonde. Il est essentiel que la planification soit axée sur le consensus, tienne compte d'un grand nombre de variables et associe tous les acteurs concernés, tout en s'appuyant sur des données fiables, car selon V. Amelung: «sans données, tout ce que je peux faire c'est deviner.»

\section{Scott C. Litin}

Pragmatique, pertinent mais aussi empreint d'humour: c'est ainsi que l'on pourrait décrire l'exposé de Scott C. Litin, médecin interniste et professeur à la Mayo Clinic de Rochester, Minnesota, qui s'est tenu peu avant la pause de midi. Sous le titre de «Making Your Next Teaching Presentation Go Better Than Your Last», il a présenté aux participants toute une série de conseils pour réussir leurs présentations.

La capacité de transmettre des contenus (de formation postgraduée, en l'occurrence) de façon à capter l'intérêt de son auditoire revêt une importance décisive dans la carrière d'un médecin. Tout d'abord pour la propre carrière du médecin formateur, mais aussi pour celle des médecins en formation, qui ont besoin d'être motivés. Lors de sa présentation, S.C. Litin a parfaitement démontré sa thèse selon laquelle ce n'est pas tant ce que l'on enseigne qui est déterminant en matière de communication, mais la manière dont on le transmet. Pour cela, il s'agit de mettre en place tout un ensemble d'éléments: une gestuelle naturelle - «comme lorsqu'on parle à des amis» - est tout aussi importante que la bonne organisation des présentations PowerPoint, tant appréciées dans les cercles de médecins (mais pas seulement). «Face the audience, slow down, use pauses, use pictures, show 
enthusiasm, tell a story, make it a performance»: voilà quelques points concrets qu'il a abordés, tout comme les recommandations suivantes: «opening statement», «limited number of points» et «strong closing». Peu de gens dans le public auraient osé contredire sa conclusion: «Your skills as a presentor are actually far more important than what you present.»

\section{Un après-midi stimulant}

L'après-midi s'est déroulé en quatre parties. Tout d'abord, les participants se sont penchés sur les perspectives de la formation médicale dans le cadre de trois séminaires. Le Prof. Pascal O. Berberat, du Centre médico-didactique pour la recherche sur la formation et l'enseignement de l'Université technique de Munich, a ensuite abordé les développements et les défis actuels en matière de formation médicale. Lors de la discussion consacrée à la politique actuelle en matière de formation médicale, animée par le Dr Iwan Rickenbacher, professeur honoraire en communication politique de l'Université de Berne, les représentants des établissements de formation postgraduée, les experts des facultés de médecine ou encore les autorités ont été invités à prendre la parole. Enfin, le président de l'ISFM a clos la journée.

Nous reviendrons ici principalement sur certains aspects abordés lors du séminaire consacré aux changements structurels dans le système de santé et visant à mettre en lumière les conséquences concrètes de ces changements sur la formation postgraduée, à commencer par les effets du nouveau financement hospitalier et ceux du système DRG. Selon un sondage mentionné par Werner Bauer, une majorité des médecins se sentent limités dans leur activité en raison de l'augmentation des tâches administratives. Les structures, les processus, les méthodes et les conditionscadres de la médecine ambulatoire et hospitalière sont soumis à de multiples changements qui influencent

\footnotetext{
ISFM-Award pour un engagement exceptionnel dans la formation postgraduée Werner Bauer a profité du premier symposium MedEd pour présenter brièvement les lauréats de I'ISFM-Award 2014 pour un engagement exceptionnel dans la formation postgraduée. Les formateurs suivants, nommés par leurs anciens médecins-assistants, ont été récompensés:

José Aguirre, Zurich; Christian Candrian, Lugano; Eva Diamantis-Karamitopoulou, Berne; Daniel Eberli, Zurich; Miodrag Filipovic, Saint-Gall; Daniel Fishman, Vaud-Valais; Josef Holtz, Zurich; Christoph Huber, Berne; Urs Hug, Lucerne; Sibylle Kohler, Zollikerberg; Martin Krause, Münsterlingen; Olaf Magerkurth, Baden; Paolo MerIani, Lugano; Silke Potthast, Schlieren; Robert Schorn, Lachen; Parham Sendi, Berne; Hervé Zender, La Chaux-de-Fonds.
}

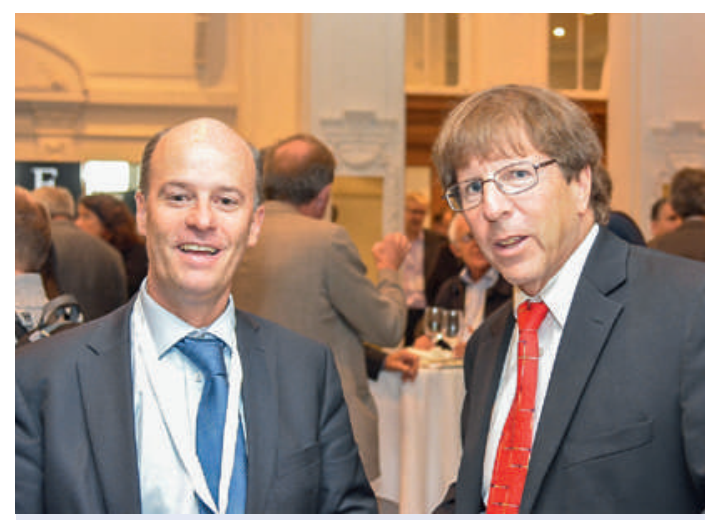

Echange entre les intervenants: Werner Kübler (à gauche) et Scott C. Litin.

également la formation médicale. A cet égard, de nombreuses questions se posent: les objectifs actuels de la formation prégraduée et postgraduée, pour la plupart quantitatifs, sont-ils toujours d'actualité? Les établissements de formation postgraduée situés en périphérie devraient-ils davantage se regrouper en réseau avec des hôpitaux universitaires? Est-il nécessaire de restructurer la formation postgraduée médicale?

Oliver Glardon, Dr en médecine vétérinaire, section Formation postgrade des professions de la santé de l'Office fédéral de la santé publique, s'est prononcé en faveur d'une conception modulaire des programmes de formation postgraduée. Selon lui, ce modèle serait notamment indiqué pour la gynécologie, discipline dans laquelle exercent de nombreuses femmes, mais également toujours plus d'hommes, qui désirent travailler à temps partiel. Le Dr Sören Huwendiek, de l'Institut d'enseignement médical à Berne, voit, quant à lui, certaines lacunes dans la formation des futurs médecins, principalement dans la communication avec les patients, l'évaluation de la capacité de travail, la gestion des erreurs, la compréhension des notions économiques fondamentales dans les différentes disciplines médicales, ainsi que dans la prise d'une anamnèse et le status clinique. Pour le Dr Christian Schirlo, responsable du décanat de la faculté de médecine de Zurich, le catalogue suisse actuel des objectifs de formation en médecine humaine est très hétérogène et complexe sur le plan de sa structure et de son contenu. Selon lui, il faudrait donc coordonner ces objectifs et les confronter aux préceptes de la psychologie de l'enseignement. Michael Jordi, secrétaire central de la Conférence suisse des directrices et directeurs cantonaux de la santé, constate, quant à lui, que les attentes face à la formation médicale prégraduée et postgraduée ont, dans l'ensemble, augmenté et que la société a le droit de savoir où ces efforts vont mener.

\section{Plaidoyer pour les humanités médicales}

Le Prof. Pascal O. Berberat, du Centre médico-didactique pour la recherche sur la formation et l'ensei- 


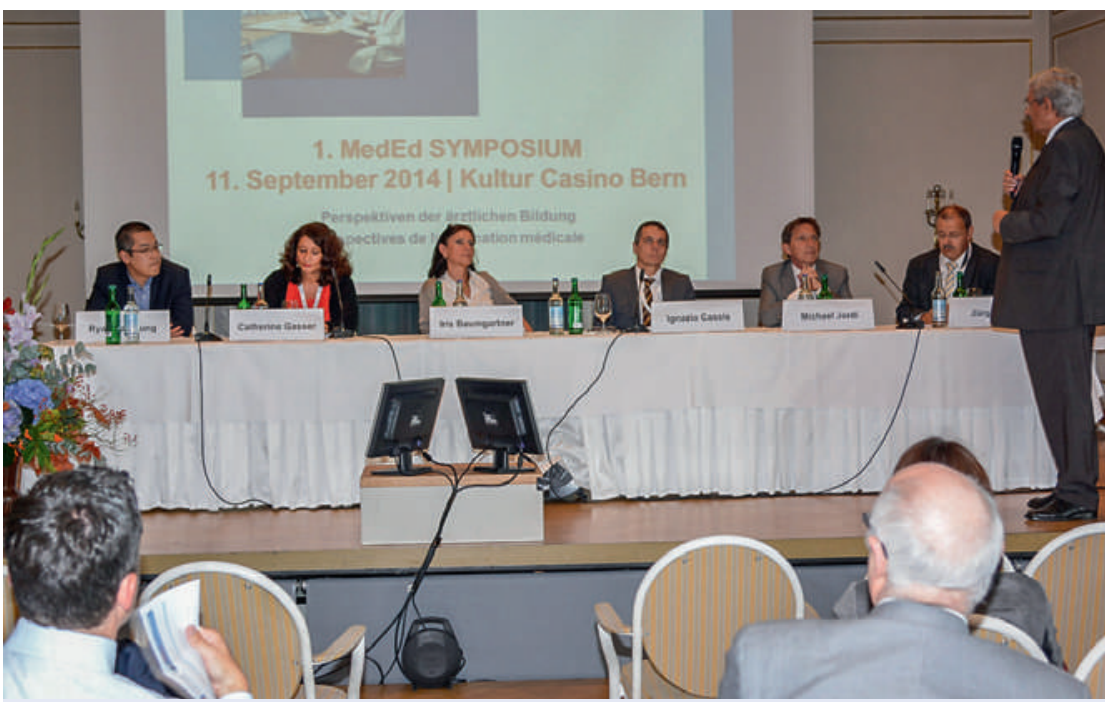

Discussion approfondie avec les représentants du corps médical, du monde politique et de l'administration. d'un feed-back régulier dans le processus d'apprentissage. Si à l'avenir, les connaissances théoriques seront de plus en plus transmises à l'aide de méthodes virtuelles, la compréhension des compétences devra s'acquérir dans le quotidien clinique. Il est donc indispensable de rendre les médecins en formation attentifs à leur rôle de modèle et à celui des formateurs. En rappelant les composantes humanistes de la médecine, à l'instar des humanités médicales, P. Berberat a affirmé que l'un des principaux objectifs de la formation prégraduée et postgraduée vise à sensibiliser les médecins en formation au fait que tous les problèmes ne peuvent pas être résolus immédiatement dans le quotidien clinique et qu'il s'agit d'apprendre à l'accepter.

\section{Discussion: envisager les défis comme une chance}

Lors de la discussion consacrée à la politique en matière de formation médicale, les participants se sont notamment intéressés aux aspects économiques. Selon la Prof. Iris Baumgartner, vice-doyenne de la formation postgrade et directrice de l'angiologie à l'Hôpital de l'Ile à Berne, la distorsion de la concurrence rend plus difficile d'accorder à la formation postgraduée la place qu'elle mérite. La formation postgraduée médicale n'est pas assez considérée car elle n'est pas suffisamment intéressante financièrement. Malgré tout, beaucoup d'argent est investi dans la formation en médecine humaine. La responsabilité du financement revient à la Confédération et aux cantons universitaires. Si davantage de moyens doivent être mis à disposition, il faudrait agir sur le plan fédéral. Le Dr Ignazio Cassis, conseiller national, suggère d'envisager les défis actuels de la formation postgraduée médicale comme une chance - bien que notre société pense avant tout en termes économiques, la formation a rarement été aussi bien soutenue qu'actuellement sur le plan financier. S'agissant de l'acquisition de connaissances théoriques et d'aptitudes pratiques, il faut avoir le courage d'utiliser les nouvelles possibilités. Catherine Gasser, responsable de la division Professions médicales à l'Office fédéral de la santé publique, ajoute que s'il est facile de générer des informations grâce aux médias numériques, il est beaucoup plus difficile d'identifier les bonnes informations et de les mettre en pratique. D'où l'importance de montrer l'exemple à la jeune génération. Pour conclure, Werner Bauer a rappelé la responsabilité de l'ISFM dans le développement cohérent de la formation postgraduée et continue médicale à l'égard de l'ensemble du domaine de la santé. 